\title{
How Grammatical Choice Shapes Media Representations of Climate (Un)certainty
}

\section{Adriana Bailey, Lorine Giangola \& Maxwell T. Boykoff}

Although mass media continue to play a key role in translating scientific uncertainty for public discourse, communicators of climate science are becoming increasingly aware of their own role in shaping scientific messages in the news. As an example of how future media research can provide relevant feedback to climate communicators, the present study examines the ways in which grammatical and word choices represent and construct uncertainty in news reporting about the Intergovernmental Panel on Climate Change (IPCC). Qualifying and hedging language and other "epistemic markers" are analyzed in four newspapers during 2001 and 2007: the New York Times and Wall Street Journal from the USA and El País and El Mundo from Spain. Though the US newspapers contained a higher density of epistemic markers and used more ambiguous grammatical constructs of uncertainty than the Spanish newspapers, all four media sources chose similar words when questioning the certainty around climate change. Moreover, the density of epistemic markers in each newspaper either remained the same or increased with time, despite ever-growing scientific agreement that human activities modify global climate. While the US newspapers increasingly adopted IPCC language to describe climate uncertainties, they also exhibited an emerging tendency to construct uncertainty by highlighting differences between IPCC reports or between scientific predictions and observations. The analysis thus helps identify articulations of uncertainty that will shape future media portrayals of climate science across varying cultural and national contexts.

Keywords: uncertainty; climate change; Intergovernmental Panel on Climate Change; IPCC; newspapers; epistemic marker

\footnotetext{
Adriana Bailey is a Ph.D. student at the Cooperative Institute for Research in Environmental Sciences, University of Colorado Boulder. Lorine Giangola is STEM Coordinator for the Graduate Teacher Program, University of Colorado Boulder. Maxwell T. Boykoff is an Assistant Professor at the Cooperative Institute for Research in Environmental Sciences, University of Colorado Boulder. Correspondence to: Adriana Bailey, CIRES, UCB 216, University of Colorado Boulder, Boulder, CO 80309, USA. Email: adriana.bailey@colorado.edu.
} 


\section{Introduction}

In its Third (TAR) and Fourth (AR4) Assessment Reports, the United Nations' Intergovernmental Panel on Climate Change (IPCC) has stated with ever-greater certainty that humans are altering Earth's climate (IPCC, 2001, 2007). Published in 2001 and 2007, respectively, the reports represent key "critical discourse moments" (Carvalho, 2005) that tether institutional actors and activities to story lines surrounding human contributions to climate change. Though the reports reflect broad scientific consensus that climate changes since the Industrial Revolution are not solely the result of natural variability in the earth system (Anderegg, Prall, Harold, \& Schneider, 2010), the media have portrayed the narratives of this discourse with varying degrees of fairness and accuracy (Boykoff, 2011).

Indeed, while scientific evidence for human-induced climate change has strengthened with time, uncertainty and disagreement have emerged as prominent themes in US mass media reporting on the topic (Antilla, 2005; Nisbet, 2009; Zehr, 2000). Possible explanations for this trend range from the macro-scale influences of a capitalist political economy to the micro-scale journalistic pressures that shape individual reporters' actions (Boykoff, 2011; Wilson, 2000). Journalists, for instance, highlight contention in order to create drama (McComas \& Shanahan, 1999; Stocking \& Holstein, 1993), providing the necessary news "hook" to justify continued coverage of climate change and win public attention for media stories (Boykoff, 2011). In climate news, they have achieved this by publicizing the arguments of a small but vocal group that contests findings that humans contribute to climate change (Boykoff, 2013; Nisbet, 2009).

Disagreement in US climate news is further accentuated by differing norms of knowledge production in science and journalism. In science, the peer-review process determines which conclusions reach print. New findings are subject to multiple reviews by experts in the relevant field of study. Although not perfect, this process nevertheless imposes protections that prevent untested or inaccurate hypotheses from entering into the ever-evolving scientific discourse. Yet rather than settling questions about knowledge, advancements in scientific understanding may complicate decisionmaking by enlarging the information pool from which diverse interpretations are developed and argued (Sarewitz, 2004). This makes uncertainties around complex scientific problems like climate change particularly susceptible to misrepresentation and manipulation (Taylor \& Buttel, 1992). The norms and standards of journalism then propel this conflict into print so that, as scientific assessments have moved toward greater convergence, US media reporting has tended to underscore controversy (Boykoff, 2007).

Whether because of distinct journalistic norms (Schudson, 2001) or distinct ideological attitudes toward climate science (Brossard, Shanahan, \& McComas, 2004), the tendency to stress uncertainty and controversy has been largely absent in news reporting in other countries. Gordon, Deines, and Havice (2010) observed that, when the Mexican newspaper Reforma discusses climate change, it focuses on the ecological impacts and consequences for developing communities rather than on scientific 
disagreement. Dotson, Jacobson, Kaid, and Carlton (2012) observed similar tendencies in Chilean newspapers. Moreover, contrarian voices are rare in Peruvian, Argentinean, and Colombian stories on global warming (Takahashi \& Meisner, 2012; Zamith, Pinto, \& Villar, 2012). In Europe, UK press have shifted focus toward potential solutions for limiting carbon emissions (Nerlich, Forsyth, \& Clarke, 2012), while German press have created an impending sense of catastrophe by translating scientific hypotheses into facts (Weingart, Engels, \& Pansegrau, 2000). Swedish media similarly underplay uncertainty as part of a conscious or unconscious effort to maintain demand for collective climate action (Olausson, 2009). These different media portrayals of climate (un)certainty are steeped in historically contingent spaces of ideology, culture, and politics, where various actors and institutions battle to shape public understanding and engagement. Through media practices and processes, in which meaning is constructed and negotiated, select portrayals gain traction over others (Boykoff, 2011; Miller \& Riechert, 2000; Nelkin, 1995), and representations of climate science, policy, and politics permeate the "everyday" (Boykoff, 2011).

Thematic-level analytical methods like critical discourse analysis (CDA), which examine texts in terms of their spatial and temporal contexts (van Dijk, 1988), provide a valuable framework for understanding how such news portrayals shape varied public understandings of climate science (Reisigl \& Wodak, 2009). CDA approaches also reveal how discursive frames privilege or marginalize particular ways of knowing (Fairclough, 1995). As Anabela Carvalho writes:

CDA allows for a richer examination of the resource used in any type of text for producing meaning. It shares with framing analysis an interest in the variable social construction of the world but puts a stronger emphasis on language and on the relation between discourse and particular social, political, and cultural contexts. (2007, p. 227)

Such qualitative methods are thus frequently used in accounting for how notions of uncertainty and risk are constructed in texts.

Once particular themes are identified, however, quantitative analyses can effectively evaluate variations in how they are presented (Miller \& Riechert, 2000), creating new opportunities for media research to provide feedback targeted at improving science communication efforts. This feedback will become increasingly important as scientists become more active in disseminating information to the public and as the changing media landscape increasingly enables direct and immediate production of science news. As an example of how future media research can provide such feedback, the present analysis departs from previous studies focused on (un)certain framing of the news and quantitatively evaluates media representations of uncertainties in climate science over time. Specifically, the analysis investigates whether US newspapers use more hedging and qualifying language than their foreign counterparts or choose fundamentally different grammatical and word choices to cast doubt on the science.

Though subtle, the distinction has implications for communication efforts. After all, some expressions of uncertainty may be more vulnerable to misinterpretation 
than others. And, despite the growing evidence linking human activity with observed climate change, the need to communicate uncertainties persists (for example, with regard to the amount of predicted temperature and sea level rise). This reality has motivated the IPCC to advocate specific grammatical structures and word choices for expressing confidence levels and probabilistic predictions clearly and consistently ("Guidance Note for Lead Authors of the IPCC Fifth Assessment Report on Consistent Treatment of Uncertainties," Mastrandrea et al., 2010).

Such guidance draws from the work of climate scientists Stephen Schneider and Richard Moss, who, after the publication of the IPCC's Second Assessment Report, set out to assess uncertainty using expert judgment. By developing a scale that paired qualitative descriptions of likelihood and quantitative probability ranges, they aimed to reduce both inadvertent and deliberate misinterpretations of uncertainty and to improve communications between the scientific community and the media, policy actors, and civil society (Schneider, 2009). The term very likely, as a result, has become associated with $90 \%$ probability or greater, while likely has come to signify $66 \%$ probability or greater (Mastrandrea et al., 2010).

Science communication strategies like that developed in the IPCC's "Guidance Note" would benefit from our knowing whether US media have adopted such specific language choices or continued communicating uncertainties in their own terms. Moreover, they would benefit from a greater understanding of how news reports use mitigating language and referencing to construct uncertainties that extend beyond those intended by the scientists. As the "Guidance Note" suggests, scientists are becoming increasingly aware of their own role in constructing and managing uncertainties in the news. Media research can therefore provide them with critical feedback about how their messages to the public are being translated and received.

To that end, the present analysis examines the grammatical and word choices used by the US prestige press to describe uncertainties related to the physical science basis of climate change. News and opinion articles were gathered from the New York Times and Wall Street Journal for 2001 and 2007, the years in which the IPCC released its TAR and AR4, respectively. These articles are contrasted with media reports from the Spanish national newspapers El País and El Mundo, which provide a necessary reference for evaluating whether US media express uncertainty more often than their foreign counterparts or use fundamentally different language to do so. While the USA has been reluctant to embrace international climate agreements, Spain has ratified the Kyoto Protocol and proposed national climate policies. Based on these ideological differences, as well as on the greater tendency to portray climate science as uncertain in the US rather than the European or Spanish-language press, we expect US news texts to employ a higher number of epistemic markers-words or expressions that communicate uncertainty-when describing climate science than Spanish news texts. We further hypothesize that ideological differences between the countries will result in distinct linguistic treatment of the uncertainties reported.

While the examination across varying cultural and political country contexts helps reveal patterns of practice across space and place, a comparison of grammatical and 
word choices across years permits us to evaluate how translations and constructions of uncertainty have evolved, not only as the evidence linking human activity and climate change has strengthened, but also as the IPCC has increasingly formalized its communication strategies for remaining "unknowns." If these events have influenced media representations of uncertainty, we expect select types of epistemic markers to have increased with time but the overall use of epistemic markers to have declined. Importantly, by tracking the evolution of uncertainty language with time, the analysis allows us to identify possible future news frames that could influence public perceptions of upcoming IPCC assessment reports.

\section{Methods}

The analysis considers newspaper articles discussing the IPCC and the climate science that forms the basis of its Working Group I (WGI) reports from the years 2001 and 2007-the years in which the IPCC published its TAR and AR4, respectively. Articles were selected from the New York Times and the somewhat more conservative Wall Street Journal in the USA and from El País and the more right-leaning El Mundo in Spain. This focus on prestige press not only permits closer comparison with previous studies (e.g. Boykoff, 2007; Brossard et al., 2004; Trumbo, 1996), but it also acknowledges the important agenda-setting role these newspapers play in mass media news coverage. Golan (2007), for instance, found a significant correlation between New York Times coverage and evening network television broadcasts, which led him to conclude that the Times sets the agenda for other media outlets. Meraz (2009) also argued that traditional US media continue to determine which stories make the news, despite the recent upswing in citizen-driven media. We assume that the Spanish national newspapers similarly shape the Spanish mass media agenda.

Newspaper articles discussing the IPCC and WGI-related science were selected from the Dow Jones news database Factiva (http://global.factiva.com). Boolean search terms for the New York Times and Wall Street Journal consisted of the following: (Intergovernmental Panel) or IPCC or I.P.C.C. or U.N. and panel same climate or (United Nations) and panel same climate or U.N. and report same climate or (United Nations) and report same climate or (International Panel on Climate). For El País and $E l$ Mundo, the following terms were considered: (Naciones Unidas) and informe w/3 (cambio climático) or ONU and (cambio climático) same informe or IPCC or (Panel Intergubernamental) or I.P.C.C. or (Grupo Intergubernamental). Identical duplicates were excluded. The search produced 152 American and 263 Spanish articles for 2007, and 33 American and 42 Spanish articles for 2001. Using 1 January 2007 as a start date, the first articles to exceed 10,000 words total were selected for each paper. Articles in which "IPCC" did not refer to the climate panel were not included in the word count. In 2001, El Mundo published only 10 articles about the IPCC, totaling 6057 words. Therefore, the first articles after 1 January 2001 to exceed 6000 words total were selected for the other three newspapers. The total number of articles analyzed, and their total word counts, are listed by year and newspaper in Table 1. 
Table 1. Number of articles and words analyzed and dates spanned for each newspaper.

\begin{tabular}{|c|c|c|c|c|c|c|c|c|}
\hline & \multicolumn{2}{|c|}{ New York Times } & \multicolumn{2}{|c|}{ Wall Street J. } & \multicolumn{2}{|c|}{ El País } & \multicolumn{2}{|c|}{ El Mundo } \\
\hline & 2001 & 2007 & 2001 & 2007 & 2001 & 2007 & 2001 & 2007 \\
\hline Articles & 8 & 11 & 7 & 11 & 14 & 15 & 10 & 16 \\
\hline Words & 6111 & 10353 & 6265 & 10208 & 6399 & 10172 & 6057 & 10313 \\
\hline Start & 5 Jan & 1 Jan & 23 Jan & 18 Jan & 23 Jan & 7 Jan & $23 \mathrm{Feb}$ & 22 Jan \\
\hline End & $19 \mathrm{Feb}$ & $4 \mathrm{Feb}$ & 11 Jun & $9 \mathrm{Feb}$ & $16 \mathrm{Jul}$ & $3 \mathrm{Feb}$ & $19 \mathrm{Jul}$ & $10 \mathrm{Feb}$ \\
\hline
\end{tabular}

Based on their context, any words or expressions suggesting room for doubteither about the physical science basis of climate change, the robustness of the IPCC assessments, or the panel's consensus or credibility-were marked as "epistemic." These included activities that produce inherently uncertain products, such as predicting, estimating, and projecting; quantitative descriptors of uncertainty, such as probabilities and likelihoods; common hedging verbs, such as believe, consider, and appear; terms questioning or criticizing the IPCC panel or report findings, such as challenge, debate, discredit, and rebut; references to those who publicly dispute climate change hypotheses in terms of contrarians, deniers, and skeptics; modifiers such as controversial, corrupt, and political, which undermine the IPCC's credibility or consensus; descriptors of likelihood, such as likely, possible, and potential; synonyms for uncertain, such as blurry, inaccurate, and speculative; and adverbial downtoners, such as almost, largely, pretty, and too. Epistemic markers also included modal verbs indicating conditionality or possibility (e.g. can, could), conjunctions introducing alternative or contingent scenarios (e.g. if, unless), numerical ranges with either one or both limits defined, and polarity markers like not.

Using a master list of epistemic markers for guidance (Appendix A, online Supplementary material), two coders analyzed 12 articles, totaling 10,097 words, from the 2007 subset. Importantly, context was always considered before a term was marked as "epistemic." Each coder was thus at liberty to mark words not included in the master list if their contextual setting indicated a degree of uncertainty, or to exclude words from the master list if their context suggested all uncertainty had been eliminated. The following examples illustrate cases in which the word uncertainty was counted (the first) and excluded (the second):

...substantial uncertainty still clouds projections of important impacts.... (New York Times, 1 January 2007)

...uncertainty was removed as to whether humans had anything to do with climate change.... (New York Times, 3 February 2007)

All epistemic markers are underlined in the examples presented throughout the text.

Context was also critical in determining whether the doubts cast were relevant to the physical science understanding of climate change, the focus of WGI. As noted by Hulme (2009), newspaper articles detailing scientific findings relevant to the IPCC's WGI commonly discuss uncertainties related to the societal impacts of climate change, including impacts to coastal cities, agriculture, ecosystems, and the spread of 
disease, or related to the costs and political difficulties of adaptation and mitigation. These topics, however, are the focus of WGII and WGIII; therefore, they are not considered in the present analysis. Common IPCC WGI topics include the link between human activities and climate change and projected changes in natural systems, which are often described in terms of temperature, precipitation, atmospheric circulation patterns, ocean acidification, ice volume, ice cover, and sea level.

A Krippendorff's (2004) alpha of 0.71 suggested a suitable level of intercoder agreement. Based on areas of disagreement, three important modifications were made to the methodology. First, because qualifying words such as almost, generally, and roughly often made statements more factually correct by acknowledging natural variability in the climate system, we opted to ignore these words unless they were used specifically to diminish certainty or confidence, as in the following example:

almost certain that ... warming is caused. (Wall Street Journal, 9 February 2007)

Second, the qualifying words about and almost were excluded when modifying numbers that had been rounded for ease of communication. One could argue that journalists introduce or enhance uncertainty by rounding numbers cited from scientific reports; however, this practice is so common in quotidian communication that we felt that counting these modifiers inflated the number of epistemic markers in the analysis.

Third, we concluded that descriptions of changes in the reports' findings and language could also construct uncertainty by suggesting inadequate levels of consensus or agreement on how climate has changed or will continue to change. An example is given below:

One reason for the growing political consensus is that the data linking fossil-fuel emissions to rising temperatures are becoming more reliable. (Wall Street Journal, 2 February 2007)

While this sentence suggests clear improvements in both the data and the level of scientific agreement, it nevertheless fails to assure readers that the IPCC has reached the necessary level of consensus, or that the data are sufficiently reliable to support the reported conclusions. Similarities and differences between these epistemic markers and the others are discussed throughout the analysis.

To evaluate how newspapers translate or construct climate uncertainty and to assess whether these translations differ between languages and over time, epistemic markers were divided among 10 grammatical categories, and word choices in each category were examined. The categories are idiomatic constructions, lexical verbs, modal verbs, verb tense, adverbs (which also included most of the polarity markers), adjectives, noun phrases, conditional clauses (i.e. clauses introduced by if, unless, whether, or variations of depending on), numerical ranges, and hedging or. Hedging ors serve much the same purpose as numerical ranges; they suggest that more than one estimate or scenario may be possible. Ors paired with whether were not counted as hedging ors since they were considered instead part of a conditional clause. The examples in bold below help illustrate each category. 
A. Bailey et al.

(1) Idiomatic constructions: "The models fall short in their representation of..." (Wall Street Journal, 9 February 2007).

(2) Lexical verbs: "The possibility of such a link has been hotly debated in recent years..." (Wall Street Journal, 2 February 2007).

(3) Modal verbs: "...Greenland could be losing more than 80 cubic miles of ice per year" (New York Times, 14 January 2007).

(4) Verb tense: “...a lo largo de este siglo, la temperatura seguiría aumentando 0,1 grados por década...” (El País, 3 February 2007).

[...over the course of this century, temperature could continue increasing 0.1 degrees [centigrade] per decade...]

(5) Adverbs: "The global warming trend does not necessarily prove that human-generated greenhouse gases are heating the planet," (New York Times, 14 January 2007).

(6) Adjectives: “...final statements are likely to go through further changes..." (New York Times, 20 January 2007).

(7) Noun phrases: "While the several dozen top models remain rough approximations ..." (New York Times, 14 January 2007).

(8) Conditional clauses: "Si la concentración de gases de efecto invernadero en la atmósfera se dobla..." (El País, 3 February 2007).

[If the concentration of greenhouse gases in the atmosphere doubles...]

(9) Numerical ranges: “...el termómetro podría elevarse por encima de los seis grados," (El Mundo, 3 February 2007).

[...the temperature could rise more than six degrees [centigrade].]

(10) Hedging or: "...warming of tropical oceans is likely to intensify such storms a percentage point or two..." (New York Times, 1 January 2007).

Differences between countries and years were tested statistically using a pooled variance Student's $t$-test. Unless otherwise stated, a $p$-value of 0.05 was used to test significance.

Epistemic markers were also categorized by tone, where negative tone was attributed to all markers that described the science as lacking, that questioned or disparaged the IPCC, that referenced doubters or contrarians, or that formed negative constructions using polarity markers, as seen in the bolded example below:

We don't know whether temperatures will continue to rise.... (Wall Street Journal, 23 January 2007)

Most remaining markers were categorized as neutral, including markers like predicting, likely, and estimate, as well as all markers belonging to the modal verbs, verb tense, conditional clauses, numerical ranges, or hedging or categories. Though under some circumstances such neutral markers are also used intentionally to construct doubt, identifying words and constructions that are clearly negative in tone gives a broadbrush sense of how often scientific uncertainties are cast in a negative light.

Uncertainty and uncertain were given their own tone classification, since these words are perceived as neutral by the scientific community and negative by the 
general public. In addition, markers referencing changes in the IPCC process or findings, partial improvements in the analysis or scientific consensus, or differences between predictions and observations (e.g. faster than [predicted/anticipated/ reported]) were each given their own independent classification. These are referred to throughout the analysis as "change," "improvement," and "surprise."

\section{Results}

USA vs. Spain

In total, 1193 epistemic markers were identified. To facilitate comparison among news sources and years, the density of epistemic makers is defined as the number of markers per 10,000 reported words. The density of epistemic markers in US newspapers significantly exceeded the density of epistemic markers in Spanish newspapers during both 2001 (189 vs. 107) and 2007 (267 vs. 136; Figure 1), supporting our prediction that the US prestige press would emphasize uncertainty over the Spanish national newspapers by using a greater number of epistemic markers. This difference is further amplified when news articles (including news briefs) are considered independently of opinion pieces for both 2001 (208 vs. 101) and 2007 (277 vs. 171). Indeed, news articles contained an equal or slightly (but not significantly) higher density of epistemic markers than opinion pieces in all four media sources during 2007. In 2001, only the US newspapers exhibited this pattern (with the difference significant for the Wall Street Journal). The US newspapers also used a higher density of epistemic markers with negative tone during both 2001 (68 vs. 13) and 2007 (72 vs. 36). This difference, though significant during both years,

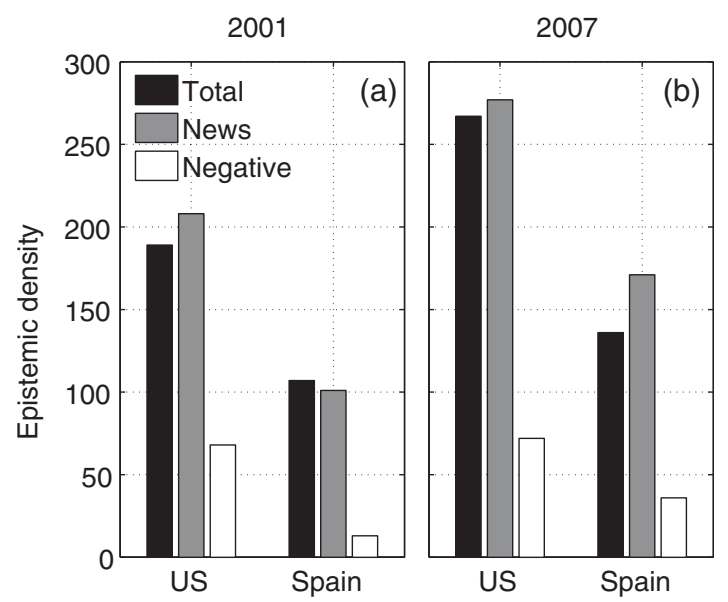

Figure 1. The epistemic density (number of epistemic markers per 10,000 reported words) of all news and opinion articles (black), the epistemic density of news articles alone (gray), and the density of epistemic markers with negative tone (white) differentiated by country for both 2001 (a) and 2007 (b). 
nevertheless narrowed with time as the Spanish newspapers increased their use of negative-tone epistemic markers.

Linguistic differences in climate reporting between the USA and Spain were identified by evaluating the percent distribution of epistemic markers across 10 grammatical categories (Figure 2). Unsurprisingly, the Spanish media sources were unique in their use of verb tense to express uncertainty (3.4\% of Spanish epistemic markers belonged to this category). Where the Spanish language uses a conditional tense to express uncertainty about future events, the English language relies on modal verbs. The percentage of epistemic markers represented by modal verbs was, consequently, higher in the US newspapers than in the Spanish (11.6\% vs. 8.8\%), although not significantly so. Combining the modal verb and verb tense categories resulted in no significant difference in relative use between the US and Spanish media sources.

More striking is the fact that the Spanish newspapers used both a higher percentage of conditional clauses and numerical ranges than the US newspapers ( $8.8 \%$ vs. $3.3 \%$ and $15.1 \%$ vs. $5.0 \%$, respectively). These epistemic markers present scientific uncertainties with no mitigation or intensification (though some translation occurs when journalists round numerical ranges for convenience). As a result, media reports that communicate uncertainties in this manner do not inflate doubts beyond the intent of the original research. Numerical ranges are also one of the types of epistemic markers that the IPCC "Guidance Note" encourages scientists to use when communicating climate science (Mastrandrea et al., 2010). Though nearly a quarter of Spanish epistemic markers were either conditional clauses or numerical ranges, less than $10 \%$ of US markers belonged to one of these grammatical categories.

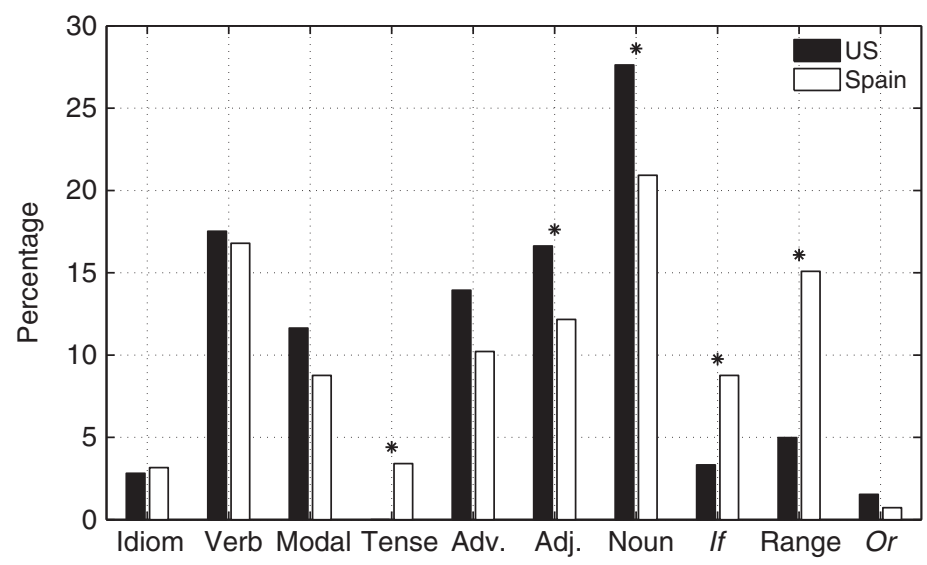

Figure 2. The percent distribution of epistemic markers across 10 grammatical categories (see methods section) for the US (black) and Spain (white) for both 2001 and 2007 combined. For example, $27.6 \%$ of US epistemic markers fell in the grammatical category noun, in comparison with $20.9 \%$ of Spanish epistemic markers. Differences between countries that are significant at the 0.05 level are marked with asterisks. 
Instead, the US media sources used a significantly higher percentage of epistemic nouns and adjectives than the Spanish media sources. These epistemic markers may cast scientific uncertainties in a negative light (e.g. epistemic markers with negative tone) or may leave the degree of uncertainty open to the reader's (mis)interpretation (e.g. hedging language). To evaluate these possibilities, both the tone and lexical choices of the epistemic nouns and adjectives were analyzed. Contrary to expectation, word choices were quite similar between the two countries.

While the percentages of epistemic noun phrases with neutral tone in the US and Spanish newspapers were $44.9 \%$ and $50.0 \%$, respectively, the percentages with negative tone were $38.4 \%$ and $39.5 \%$, respectively. Neutral epistemic nouns included words like probabilities, likelihoods, approximations, estimates, projections, and ranges. Meanwhile, negative-tone noun phrases tended to frame climate change as a hoax, controversy, and debate, call attention to skeptics of the science and flaws in the report, and refer to the IPCC process using gerunds like squabbling and shouting. Of the epistemic nouns with negative tone, the majority in both countries referred to disagreement, debate, or climate "skepticism," all of which suggest a lack of scientific consensus around climate change. Specifically, $56.6 \%$ and $64.7 \%$ of the negative-tone epistemic noun phrases from the US and Spanish newspapers, respectively, negated consensus. The following are examples of such markers:

...negotiations on a worldwide treaty to reduce global warming collapsed in part because of disagreements over the role of natural ecosystems like forests in sopping up carbon dioxide from the atmosphere.... (New York Times, 11 January 2001) While everyone concedes that the Earth is about a degree Celsius warmer than it was a century ago, the debate continues over the cause and consequences. (Wall Street Journal, 5 February 2007)

In total, $8.6 \%$ of the epistemic noun phrases describing climate change included the word debate, a word that connotes a politicized rather than scientific process. Finally, though the noun uncertainty is particularly vulnerable to misinterpretation due to its different interpretations among scientists and the public, there was no significant difference in relative usage between the US and Spanish media sources. Uncertainty (incertidumbre in Spanish) comprised $11.6 \%$ of the US and $7.0 \%$ of the Spanish epistemic nouns, and in only one example from each country were the uncertainties explicitly described as having been reduced.

As with noun phrases, adjectival lexical choices were also similar between countries. About a quarter of US and Spanish epistemic adjectives $(26.9 \%$ and $24.0 \%$, respectively) were represented by the words likely and probable (the latter also being the Spanish translation for likely). The slightly more mitigating possible and potential constituted $9.2 \%$ of the US epistemic adjectives and $10.0 \%$ of the Spanish, while uncertain and its synonyms comprised less than $5 \%$ of the epistemic adjectives from each country (3.8\% and $4.0 \%$, respectively). Past predicates of neutral-tone verbs like predict formed $8.5 \%$ of the US and $4.0 \%$ of the Spanish epistemic adjectives, while a third of the epistemic adjectives from each country were grouped as "other" $31.5 \%$ and $30.0 \%$ for the USA and Spain, respectively). Modifiers belonging to the "other" 
grouping were mostly negative in tone. They included words like inaccurate, incomplete, flawed, and unpredictable:

[President George Bush] cited 'the incomplete state of scientific knowledge' about global climate change. (Wall Street Journal, 22 March 2001)

The remaining epistemic adjectives belonged to the three categories of "change," "improvement," and "surprise." As described in the methods section, these markers construct uncertainty by presenting (1) disparate results with no background information for why such discrepancies exist ("change"), (2) partial progress in data, models, or knowledge that suggest a need for continued improvement ("improvement"), or (3) observations that have differed from predictions ("surprise"). A significantly higher percentage of Spanish epistemic adjectives highlighted "changes" compared with US adjectives (12.0\% vs. $2.3 \%)$. In contrast, the US newspapers were significantly more likely than the Spanish newspapers to construct uncertainty with adjectives connoting "surprises" (4.6\% vs. 0.0\%). Although Spanish epistemic adjectives were more likely than US epistemic adjectives to describe "improvements" that reduce uncertainties (12.0\% vs. $7.7 \%)$, this difference was not statistically significant.

\section{1 vs. 2007}

Though the scientific community substantially improved its understanding of climate change and strengthened the link between human activities and warming in the years spanning the TAR and the AR4, the overall density of epistemic markers in climate news either remained the same-as observed for the Wall Street Journal (236 vs. 235) and El País (131 vs. 139)-or significantly increased with time-as observed for the New York Times (141 vs. 297) and El Mundo (81 vs. 133; Figure 3). When news articles are considered in isolation, the density of epistemic markers also increased significantly in El País (125 vs. 176). This finding does not suggest that newspapers failed to communicate improvements in scientific understanding. Rather, as they did so, they also dedicated more word space to remaining uncertainties about the science and about the panel responsible for synthesizing climate information.

In those media sources in which the density of epistemic markers increased, so too did the use of markers with negative tone. While negative-tone markers made up $12.8 \%$ and $8.2 \%$ of the total epistemic markers in the New York Times and El Mundo, respectively, in 2001, they comprised $23.7 \%$ and $35.0 \%$ of the total markers in each paper in 2007. These significant increases in the percentages of markers with negative tone suggest a real shift toward more negative language rather than a simple increase in overall hedging. The Wall Street Journal was the one paper in which the percentage of markers with negative tone decreased with time, from $49.3 \%$ of the paper's epistemic markers in 2001 to $31.7 \%$ in 2007. Such results suggest that diachronic changes in epistemic density and tone tend to blur broad-brush differences in climate reporting between the USA and Spain. 


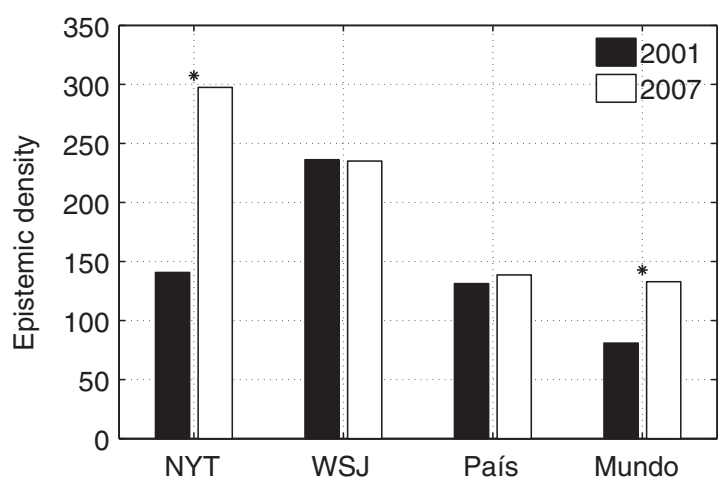

Figure 3. Total epistemic density in 2001 (black) and 2007 (white) for the four newspapers: New York Times (NYT), Wall Street Journal (WSJ), El País (País), and El Mundo (Mundo). Differences in time that are significant at the 0.05 level are marked with asterisks.

Indeed, though the US newspapers exhibited a significantly higher density of epistemic markers with negative tone in both years, the Spanish newspapers showed a significant increase in the density of negative-tone markers between 2001 and 2007 (13 vs. 36). In particular, the percentage of Spanish epistemic nouns indicating debate, disagreement, and skepticism increased from $12.5 \%$ to $30.6 \%$, a significant increase at the 0.10 level. While the Spanish media reports from 2001 did not use the word debate once in describing the IPCC or global warming, the epistemic density of this word rose to 4 in 2007, a significant increase at the 0.05 level. Changes in Spanish epistemic markers with time therefore indicate more questioning of climate science and scientific consensus.

In contrast, changes in US epistemic markers suggest potential improvements in the linguistic treatment of climate uncertainty, including increased adoption of IPCC language choices. Use of the adjective likely, for instance, more than tripled from 2001 to 2007 (Figure 4). In comparison, use of the similar but more mitigating words possible and potential did not change with time, a finding that suggests the adoption of likely was indeed intentional. This inference is further supported by the use of quotes in the following example:

In its last report, in 2001, the panel, consisting of hundreds of scientists and reviewers, said the confidence level for its projections was 'likely,' or 60 to 90 percent. (New York Times, 3 February 2007)

Use of numerical ranges, which are also promoted by the IPCC "Guidance Note" (Mastrandrea et al., 2010), similarly increased in the US newspapers (Figure 4).

In addition to adopting IPCC-recommended language, there is evidence that the US newspapers were increasingly aware of climate science improvements in 2007. Epistemic markers suggesting partial progress in data, models, and understanding ("improvement") increased significantly with time (Figure 5). So too did explicit descriptions of reductions in uncertainty, which the following example illustrates: 


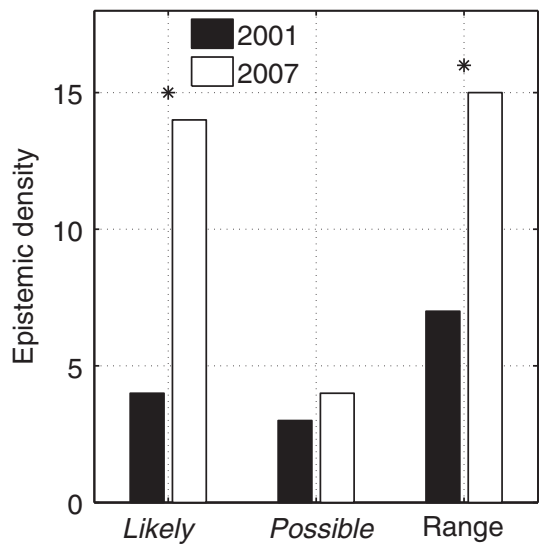

Figure 4. From left to right, the epistemic densities of variations of the adjective likely, the words possible and potential, and numerical ranges in the US newspapers for 2001 (black) and 2007 (white). Differences in time that are significant at the 0.05 level are marked with asterisks.

The new document contains 'a strengthening of findings, a narrowing of uncertainty....' (Wall Street Journal, 2 February 2007)

Nevertheless, the US newspapers simultaneously constructed greater climate uncertainty in 2007 by highlighting more "changes" and "surprises" in their news reports (Figure 5). In some cases, US media used these epistemic markers to underscore the urgency of the environmental problem:

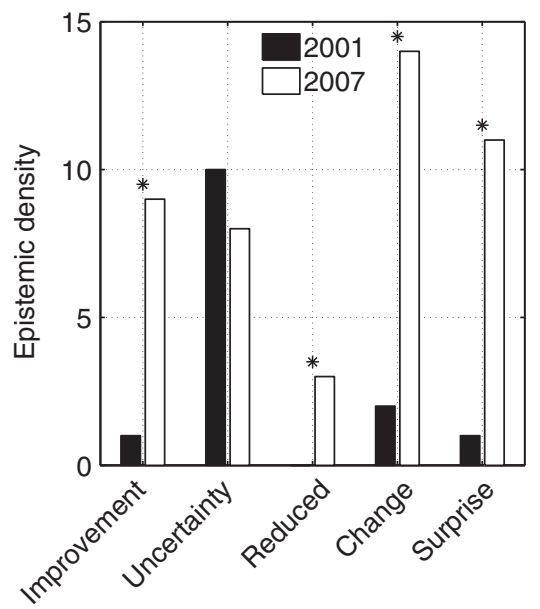

Figure 5. From left to right, the epistemic densities of markers suggesting partial "improvement," markers composed of variations of uncertain or uncertainty, markers suggesting reductions in uncertainty, markers referencing changes in IPCC reports ("change"), and markers referencing differences between predictions and observations ("surprise") for the US newspapers in 2001 (black) and 2007 (white). Differences in time that are significant at the 0.05 level are marked with asterisks. 
Ice in arctic seas also is melting faster than expected. (Wall Street Journal, 9 February 2007)

In other cases, they clearly intended to discredit climate science:

And according to people who have seen that draft, it contains startling revisions of previous U.N. predictions. (Wall Street Journal, 5 February 2007)

Regardless of their intention, by presenting side-by-side comparisons of past IPCC conclusions and either new findings or contrasting observations, the US newspapers created an apparent sense of discrepancy. Readers lacking the background information necessary to understand these seeming discrepancies could have interpreted them as indications of uncertain science.

\section{Discussion and Conclusions}

Uncertainty is an inherent feature of contemporary scientific inquiry as well as everyday decision-making. Yet "translating error bars into ordinary language," as Henry Pollack described it (2003, p. 77), may result in varied representations of uncertainty with different potentials to inform individual and collective action, particularly since the reduction of uncertainty around scientific issues has long been framed as a prerequisite for meaningful political and policy progress (Zehr, 2000). This mindset may stem from commonly held reductionist views that uncertainties are:

due to an incomplete definition of an essentially determinate cause-effect system ... they suggest that the route to better control of risks is more intense scientific knowledge of that system, to narrow the supposed uncertainties and gain a more precise definition of it. (Wynne, 1992, p. 116)

According to Hulme, such thinking has guided climate science policy considerations through "simple linear" or "bi-polar" framing, which claims that "either the scientific evidence is strong enough for action or else it is too weak for action" (2010, p. 23). In this context, understanding diverse characterizations of uncertainty in public discourse is critical for elucidating potential barriers to informed policy discussions and appropriate climate action.

Through an analysis of US and Spanish newspapers, this study has evaluated the degree to which mass media directly report the caveats and errors associated with climate assessments or perpetuate the notion of "uncertain science" that has often framed climate news in the USA (Antilla, 2005; Boykoff, 2007; Zehr, 2000). In particular, by characterizing the grammatical constructions and lexical choices used by the media to represent climate unknowns, we have evaluated whether expressions of climate uncertainty are fundamentally different across languages and cultures and whether these expressions have evolved with time, in response to scientific progress and new communication strategies. This approach provides important feedback for climate communicators, like the IPCC, who wish to express remaining uncertainties clearly and consistently in order to support well-informed decision-making around the world. 
Differences in epistemic markers across cultures and over time were identified by considering news and opinion articles from two US and two Spanish national newspapers for the years 2001 and 2007. Given the distinct ideological viewpoints historically expressed by the US and Spain on climate policy, as well as the relationship between political ideology and framing of climate news (Brossard et al., 2004; Carvalho, 2007; Ereaut \& Segnit, 2006), we expected the US newspapers to hedge more than the Spanish newspapers, using grammatical constructs and lexical choices that express uncertainty more negatively and/or ambiguously. The overall density of epistemic markers and the density of epistemic markers with negative tone were indeed higher in the US newspapers during both 2001 and 2007. In addition, while the Spanish newspapers were more likely to report scientific uncertainties using numerical ranges and conditional clauses-some of the least ambiguous expressions of uncertainty-the US newspapers showed a greater tendency to use noun phrases or adjectives to translate or construct uncertainty around climate change. Lexical choices within specific grammatical categories, however, were remarkably similar across the two languages, which suggests similar vocabularies are used in both cultures to represent uncertainty in public discourse.

We also hypothesized that if the IPCC's communication efforts had influenced news reporting as the scientific understanding of climate change advanced, then select expressions of uncertainty should have become more prevalent while the overall density of epistemic markers decreased with time. Though the use of numerical ranges and the adjective likely did increase between 2001 and 2007-indications that intentional scientific communication strategies are shaping media reporting-the overall density of epistemic markers did not decrease significantly in either US paper. Ignoring the small increase in markers describing reduced uncertainty or partial progress in data, models, or predictions ("improvement") does not change this result.

An important direction for future research is to determine why US climate news continued to employ mitigating language with such frequency, despite everstrengthening scientific understanding of and consensus around climate change. One possibility is that news reports reflect a natural tendency to hedge scientific information. Consequently, the more scientific information contained in a single article, the higher the epistemic density. It would be useful to consider whether this hedging tendency is ubiquitous across science disciplines or unique to the field of climate science. Another possibility is that politicized attacks on climate science throughout the 1990s and 2000s have resulted in a more cautious presentation of new scientific results by journalists. The influence of contrarians in shaping climate news appears evident in that the two Spanish newspapers referred more frequently to deniers, disagreement, and debate in 2007 than in 2001. Tracking the influence of contrarian arguments on climate reporting would be another important direction for future research and one that would provide valuable feedback to climate communication efforts. Corollary studies of media treatment of uncertainty around agricultural biotechnology (e.g. Besley \& Shanahan, 2005), posited links between autism and vaccines (e.g. Dixon \& Clarke, 2012), and other science and environment issues cast 
as controversial (Friedman, Dunwoody, \& Rogers, 1999) provide insights into ongoing examinations along these research trajectories.

An unexpected result of the analysis was that even as the US newspapers modified their linguistic treatment of climate change to reflect scientific advancements and IPCC language choices, they simultaneously constructed new uncertainty by highlighting "changes" and "surprises" with greater frequency. "Change" markers, which described differences between IPCC assessments, became so prominent that they defined the news hooks for at least one New York Times news article and one Wall Street Journal opinion piece in 2007. The following are excerpts from their respective lead paragraphs:

In Paris today the panel will issue its fourth assessment, and people familiar with its deliberations say it will moderate its gloom on sea level rise, lowering its worstcase estimate. (New York Times, 2 February 2007)

Yet the real news in the fourth assessment from the Intergovernmental Panel on Climate Change (IPCC) may be how far it is backpedaling on some key issues. (Wall Street Journal, 5 February 2007)

As research on various dimensions of the science of climate change advances (e.g. detection and attribution, drivers of climate change, climate sensitivity), and newspapers struggle to find hooks that maintain public interest in this issue, our analysis suggests news stories will continue to feature "change" and "surprise" epistemic markers in ongoing coverage. It therefore behooves climate science communicators to consider how providing news consumers with background information about apparent discrepancies in scientific findings can diffuse public perceptions of uncertainty (Corbett \& Durfee, 2004) and inform climate policy discussions. Our findings demonstrate that emerging and waning linguistic patterns can subtly reframe climate science discourse in the public arena. Interdisciplinary research endeavors like this can provide vitally important insights into the relative successes of communication strategies in the twenty-first century.

\section{Acknowledgments}

Thanks to Javier Rivas of the University of Colorado Boulder for inspirational discussions on epistemic markers in Spanish and English. Adriana Bailey's work was supported by a Graduate Research Fellowship from the Cooperative Institute for Research in Environmental Sciences, University of Colorado Boulder.

\section{References}

Anderegg, W. R. L., Prall, J. W., Harold, J., \& Schneider, S. H. (2010). Expert credibility in climate change. Proceedings of the National Academy of Sciences, 107, 12107-12109. doi:10.1073/ pnas. 1003187107

Antilla, L. (2005). Climate of scepticism: US newspaper coverage of the science of climate change. Global Environmental Change, 15, 338-352. doi:10.1016/j.gloenvcha.2005.08.003

Besley, J. C., \& Shanahan, J. (2005). Media attention and exposure in relation to support for agricultural biotechnology. Science Communication, 26, 347-367. doi:10.1177/1075547005 275443 
Boykoff, M. T. (2007). From convergence to contention: United States mass media representations of anthropogenic climate change science. Transactions of the Institute of British Geographers, 32, 477-489. doi:10.1111/j.1475-5661.2007.00270.x

Boykoff, M. T. (2011). Who speaks for climate? Making sense of mass media reporting on climate change. Cambridge and New York: Cambridge University Press.

Boykoff, M. (2013). Public enemy no. 1? Understanding media representations of outlier views on climate change. American Behavioral Scientist, 57, 796-817. doi:10.1177/0002764213476846

Brossard, D., Shanahan, J., \& McComas, K. (2004). Are issue-cycles culturally constructed? A comparison of French and American coverage of global environmental change. Mass Communication \& Society, 7, 359-377. doi:10.1207/s15327825mcs0703_6

Carvalho, A. (2005). Representing the politics of the greenhouse effect. Critical Discourse Studies, 2, 1-29. doi:10.1080/17405900500052143

Carvalho, A. (2007). Ideological cultures and media discourses on scientific knowledge: Re-reading news on climate change. Public Understanding of Science, 16, 223-243.

Corbett, J. B., \& Durfee, J. L. (2004). Testing public (un)certainty of science: Media representations of global warming. Science Communication, 26, 129-151. doi:10.1177/1075547004270234

Dixon, G. C., \& Clarke, C. E. (2012). Heightening uncertainty around certain science: Media coverage, false balance, and the autism-vaccine controversy. Science Communication, 35, 358-382. doi:10.1177/1075547012458290

Dotson, M. D., Jacobson, S. K., Kaid, L. L., \& Carlton, J. S. (2012). Media coverage of climate change in Chile: A content analysis of conservative and liberal newspapers. Environmental Communication, 6, 64-81.

Ereaut, G., \& Segnit, N. (2006). Warm words. How are we telling the climate story and can we tell it better? Retrieved from http://www.ippr.org

Fairclough, N. (1995). Media discourse. London: Edward Arnold.

Friedman, S. M., Dunwoody, S., \& Rogers, C. L. (Eds.). (1999). Communicating uncertainty: Media coverage of new and controversial science. New York: Routledge.

Golan, G. (2007). Inter-media agenda setting and global news coverage. Journalism Studies, 7, 323-333. doi:10.1080/14616700500533643

Gordon, J. C., Deines, T., \& Havice, J. (2010). Global warming coverage in the media: Trends in a Mexico City newspaper. Science Communication, 32, 143-170. doi:10.1177/1075547009340336

Hulme, M. (2009). Mediating the messages about climate change: Reporting the IPCC fourth assessment in the UK print media. In T. Boyce \& J. Lewis (Eds.), Climate change and the media (pp. 117-128). New York: Peter Lang.

Hulme, M. (2010, November 17). The year climate science was redefined. The Guardian, p. 23.

IPCC. (2001). Summary for policymakers. In J. T. Houghton, Y. Ding, D. J. Griggs, M. Noguer, P. J. van der Linden, X. Dai, ... C. A. Johnson (Eds.), Climate change 2001: The scientific basis. Contribution of working group I to the third assessment report of the intergovernmental panel on climate change (pp. 1-20). Cambridge and New York: Cambridge University Press.

IPCC. (2007). Summary for policymakers. In S. Solomon, D. Qin, M. Manning, Z. Chen, M. Marquis, K. B. Averyt, ... H. L. Miller (Eds.), Climate change 2007: The physical science basis: Contribution of working group I to the fourth assessment report of the intergovernmental panel on climate change (pp. 1-18). Cambridge and New York: Cambridge University Press.

Krippendorff, K. (2004). Content analysis: An introduction to its methodology. Thousand Oaks, CA: Sage.

Mastrandrea, M. D., Field, C. B., Stocker, T. F., Edenhofer, O., Ebi, K. L., Frame, D. J., ... Zwiers, F. W. (2010). Guidance note for lead authors of the IPCC fifth assessment report on consistent treatment of uncertainties. Retrieved from http://www.ipcc.ch

McComas, K., \& Shanahan, J. (1999). Telling stories about global climate change: Measuring the impact of narratives on issue cycles. Communication Research, 26, 30-57. doi:10.1177/ 009365099026001003 
Meraz, S. (2009). Is there an elite hold? Traditional media to social media agenda setting influence in blog networks. Journal of Computer-Mediated Communication, 14, 682-707. doi:10.1111/ j.1083-6101.2009.01458.x

Miller, M. M., \& Riechert, B. P. (2000). Interest group strategies and journalistic norms. In S. Allan, B. Adam, \& C. Carter (Eds.), Environmental risks and the media (pp. 45-54). London and New York: Routledge.

Nelkin, D. (1995). Selling science. New York, NY: W.H. Freeman.

Nerlich, B., Forsyth, R., \& Clarke, D. (2012). Climate in the news: How differences in media discourse between the US and UK reflect national priorities. Environmental Communication, 6, 44-63.

Nisbet, M. C. (2009). Communicating climate change: Why frames matter for public engagement. Environment: Science and Policy for Sustainable Development, 51, 12-23. doi:10.3200/ ENVT.51.2.12-23

Olausson, U. (2009). Global warming - Global responsibility? Media frames of collective action and scientific uncertainty. Public Understanding of Science, 18, 421-436. doi:10.1177/09636625070 81242

Pollack, H. (2003). Can the media help science? Skeptic, 102, 73-80.

Reisigl, M., \& Wodak, R. (2009). The Discourse-Historical Approach (DHA). In R. Wodak \& M. Meyer (Eds.), Methods for critical discourse analysis (pp. 87-121). London: Sage.

Sarewitz, D. (2004). How science makes environmental controversies worse. Environmental Science and Policy, 7, 385-403. doi:10.1016/j.envsci.2004.06.001

Schneider, S. (2009). Science as a contact sport: Inside the battle to save earth's climate. Washington, DC: National Geographic.

Schudson, M. (2001). The objectivity norm in American journalism. Journalism, 2, 149-170. doi:10.1177/146488490100200201

Stocking, S. H., \& Holstein, L. W. (1993). Constructing and reconstructing scientific ignorance: Ignorance claims in science and journalism. Science Communication, 15, 186-210. doi:10.1177/107554709301500205

Takahashi, B., \& Meisner, M. (2012). Climate change in Peruvian newspapers: The role of foreign voices in a context of vulnerability. Public Understanding of Science, 1, 1-16. doi:10.1177/ 0963662511431204

Taylor, P., \& Buttel, F. (1992). How do we know we have global environmental problems? Science and the globalization of environmental discourse. Geoforum, 23, 405-416. doi:10.1016/00167185(92)90051-5

Trumbo, C. (1996). Constructing climate change: Claims and frames in US news coverage of an environmental issue. Public Understanding of Science, 5, 269-283. doi:10.1088/0963-6625/5/ $3 / 006$

Van Dijk, T. A. (1988). News as discourse. Hillsdale: Lawrence Erlbaum.

Weingart, P., Engels, A., \& Pansegrau, P. (2000). Risks of communication: Discourses on climate change in science, politics, and the mass media. Public Understanding of Science, 9, 261-283. doi:10.1088/0963-6625/9/3/304

Wilson, K. M. (2000). Drought, debate, and uncertainty: Measuring reporters' knowledge and ignorance about climate change. Public Understanding of Science, 9, 1-13. doi:10.1088/0963$6625 / 9 / 1 / 301$

Wynne, B. (1992). Uncertainty and environmental learning: Reconceiving science and policy in the preventative paradigm. Global Environmental Change, 2, 111-127. doi:10.1016/0959-3780 (92)90017-2

Zamith, R., Pinto, J., \& Villar, M. E. (2012). Constructing climate change in the Americas: An analysis of news coverage in U.S. and South America newspapers. Science Communication, 35, 334-357. doi:10.1177/1075547012457470

Zehr, S. C. (2000). Public representations of scientific uncertainty about global climate change. Public Understanding of Science, 9, 85-103. doi:10.1088/0963-6625/9/2/301 